\title{
The Use of Metamers to Compare the Color Vision of Observers
}

\section{J. M. Ezquerro, ${ }^{1 *}$ F. Carreño, ${ }^{1}$ J. M. Zoido, ${ }^{1}$ E. Bernabeu ${ }^{2}$}

\author{
${ }^{1}$ Escuela Universitaria de Optica, Universidad Complutense de Madrid, C/Arcos de Jalón s/n, Madrid 28037, Spain \\ ${ }^{2}$ Facultad de CC. Físicas, Universidad Complutense de Madrid, Ciudad Unversitaria s/n, Madrid 28040, Spain
}

Received 18 September 1999; accepted 1 July 2000

\begin{abstract}
In this research we compare the colorimetric behavior of several observers. For color centers recommended by CIE we have produced large sets of spectral distributions, which are metameric for the CIE 1931 standard observer. For each one of the color centers, we compare the clouds of chromaticity coordinates with the chromaticity thresholds. We define a parameter that provides a quantitative measure of the interobserver variability. This parameter is used to arrange the observers by their degree of likeness. A similar procedure has been used to compare two real observers. It is shown how there is no reciprocity between the colorimetric behavior of two real observers. () 2001 John Wiley \& Sons, Inc. Col Res Appl, 26, 262-269, 2001
\end{abstract}

Key words: color vision; colorimetry

\section{INTRODUCTION}

The colorimetric behavior of different observers is an important area of research in color science. Several studies have been carried out in order to verify the suitability of CIE 1931 Standard Observer ${ }^{1-6}$ and CIE 1964 standard observer. ${ }^{7-9}$ The design of adequate commercial devices requires us to evaluate the differences among the behavior of different color detection, or color reproduction, systems. Thus, there is no doubt about the interest in obtaining a quantitative measure of the differences in color performance between a given observer, or device, and a standard one.

A usual procedure when comparing two observers is to analyze the similarity of their corresponding sets of colormatching functions (cmf's). This comparison can also be carried out by studying the differences among the tristimu-

\footnotetext{
* Correspondence to: Dr. J. M. Ezquerro, Escuela Universitaria de Optica, Universidad Complutense de Madrid, C/Arcos de Jalón s/n, E-28037 Madrid, España (e-mail: ferpo@fis.ucm.es) (C) 2001 John Wiley \& Sons, Inc.
}

lus values associated with each observer when considering a fixed set of color centers (see for instance Refs. 5, 10, 11). It is well known that, due to interobserver variability, a given physical stimulus evokes different color sensations in each observer.

The conclusions derived from the study of two observers depend on the particular method used when analyzing the data. In this work, we propose a procedure to compare the colorimetric behavior of two observers. The method proposed by us can be applied in a systematic way when different observers are considered.

We present the proposed method in Section II. In Section III, we provide the results obtained for different sets of cmf's. We also provide criteria to establish an order of likeliness among the different observers under consideration. Section IV is devoted to analyzing a special case of two observers for whom the cmf's and the chromaticity thresholds have been determined. ${ }^{10}$ Finally, the conclusions are given in Section V.

\section{OBSERVERS AND COLOR-MATCHING FUNCTIONS}

Let $\left\{x_{i}^{k}(\lambda)\right\}(i=1,2,3)$ be the set of cmf's that determine the colorimetric behavior of a real observer $k$. The tristimulus values associated with a spectral radiant flux $\rho(\lambda)$ are given by

$$
X_{i}^{k}=K \int_{\lambda_{1}}^{\lambda_{2}} \rho(\lambda) x_{i}^{k}(\lambda) d \lambda, \quad(i=1,2,3),
$$

with $K$ being a constant that depends on the system of primary stimuli used to specify the cmf's, and $I=\left[\lambda_{1}, \lambda_{2}\right]$ is the spectral range considered. The spectral range is taken as $I=[360,830] \mathrm{nm}$ in the case of the CIE 1931 standard observer. ${ }^{13}$ In general, the interval $I$ depends on the spectral 
range considered when measuring the set $\left\{x_{i}^{k}(\lambda)\right\}$. Most authors take $\lambda_{1}=400 \mathrm{~nm}$ and $\lambda_{2}=700 \mathrm{~nm}$.

Any color stimulus perceived by a given observer $k$ can be specified in the corresponding color-representation system as a point defined by the vector $\mathbf{X}^{k}=\left(X_{1}^{k}, X_{2}^{k}, X_{3}^{k}\right)$. Thus, the set $R^{k}=\left\{\mathbf{X}^{k}\right\}$ defined by the tristimulus values of all possible color stimuli provides the region that contains all the possible color sensations perceived by the observer $k$. We refer to the subset $R^{k}$ as color-representation system associated with the observer $k$.

In Eq. (1), cmf's are specified with regard to a set of primary stimuli $\left\{\mathbf{C}_{\mathbf{i}}\right\}$. If we consider another set of primary stimuli $\left\{\mathbf{C}_{\mathbf{i}}^{\prime}\right\}$ linearly related with the previous one, i.e., $\mathbf{C}_{\mathbf{m}}^{\prime}=\sum_{n=1}^{3} c_{n m} \mathbf{C}_{\mathbf{n}}$, the relation between the corresponding tristimulus values, $\mathbf{X}=\left(X_{1}, X_{2}, X_{3}\right)$ and $\mathbf{X}^{\prime}=\left(X_{1}^{\prime}, X_{2}^{\prime}\right.$, $\left.X_{3}^{\prime}\right)$, is given by

$$
\mathbf{X}=C \mathbf{X}^{\prime},
$$

with $C=\left\{c_{m n}\right\}$.

In general, the set of cmf's of a real observer, $\left\{x_{i}^{k}(\lambda)\right\}$, differs from the set $\left\{x_{i}^{s o}(\lambda)\right\}$ associated with the standard one. Thus, Eq. (1) provides different tristimulus values when considering both cases. A real observer and a standard one generate different color-representation systems.

Let us consider an ensemble of $Q$ observers, each one of them being characterized by its corresponding set of cmf's $\left\{x_{i}^{k}(\lambda)\right\}$ ( $k$ running from 1 to $Q$ and $i$ from 1 to 3 ). We take one of them as the reference observer: CIE 1931 Standard Observer in our case. The interobserver variability can be estimated by analyzing the differences in the perception of a given set of $R$ reference stimuli. For each one of these color stimuli, we have produced a set of $S$ spectral radiant power distributions, which are metameric for the reference observer.

Let $\rho_{j}^{l}(\lambda)$ be the $j$-th metameric spectral radiant power distribution associated with the $l$-th reference stimulus, where $l$ runs from 1 to $R$ and $j$ runs from 1 to $S$. For the $k$-th observer, distribution $\rho_{j}^{l}(\lambda)$ produces the tristimulus values

$$
\begin{aligned}
X_{i, j}^{k, l} & =K \int_{\lambda_{1}}^{\lambda_{2}} x_{i}^{k}(\lambda) \rho_{j}^{l}(\lambda) d \lambda, \\
i & =1,2,3, \\
j & =1, \ldots, S, \\
k & =1, \ldots, Q, \\
l & =1, \ldots, R .
\end{aligned}
$$

For a distribution $\rho_{j}^{l}(\lambda)$, it is expected that, if the set $\left\{x_{i}^{k}(\lambda)\right\}$ does not differ strongly from the set $\left\{x_{i}^{s o}(\lambda)\right\}$, Eq. (3) should provide similar results for both sets. In this case, the observer $k$ has a colorimetric behavior similar to that of the reference observer. In the following analysis, we try to elucidate some conclusion about this subject.

By using the set of cmf's $\left\{x_{i}^{k}(\lambda)\right\}$, we have computed the chromaticity coordinates for the $S$ metameric distributions associated with color center $l$. Due to interobserver vari- ability, each spectral distribution $\rho_{j}^{l}(\lambda)$ produces a different point in the chromaticity diagram. In this way, we obtain a cloud of points. If the behavior of both observers is similar, this cloud should be close to the point defined by the chromaticity coordinates perceived by the standard observer for the color center under consideration. To check this closeness, we compare the cloud of points with the chromaticity threshold experimentally determined for the observer $k$. If the distribution of points is inside the threshold, we could conclude that there is no difference between the colorimetric behavior of both observers for the considered color center. If the cloud of points is outside the threshold, the color sensation perceived by each observer differs. This point was noted by Hita et al. ${ }^{14}$ when considering what they called "weak definition of metamerism." To quantify this difference, we compute the distance $d_{a}^{k, l}$ from $\mathbf{x}^{\mathbf{k}, \mathbf{l}}$ to $\overline{\mathbf{x}}^{\mathbf{k}, \mathbf{l}}, \mathbf{x}^{\mathbf{k}, \mathbf{l}}$ being the center of the threshold and $\overline{\mathbf{x}}^{\mathbf{k}, \mathbf{l}}$ being the point defined as the average chromaticity coordinates of the cloud of points. The straight line joining both points intersects the ellipse representing the threshold at point $\mathbf{x}_{\mathbf{t h}}^{\mathbf{k}, \mathbf{l}}$. The distance from this point to the center of the threshold is labeled as $d_{t h}^{k, l}$. In this case, quotient,

$$
\Omega^{k, l}=\frac{d_{a}^{k, l}}{d_{t h}^{k, l}}
$$

could provide a quantitative measure of interobserver variability when considering the $l$-th color center.

\section{OBSERVERS METAMERISM AND COLOR-DIFFERENCE THRESHOLDS}

We have considered in this research the following sets of cmf's, all of them determined for a visual field of $2^{\circ}$ :

- The mean observer obtained by averaging, SBM, the ten sets of cmf's provided by Stiles-Burch ${ }^{15}$ labeled as $S B i$ (where $i$ runs from 1 to 10).

- Standard observer CIE 1931 modified by VOS. ${ }^{16}$

- Observers MM, JAM and CF.10,11

Some of these sets of cmf's take negative values when they are specified in the CIE 1931 system of primary stimuli. To avoid the possible difficulties derived from this fact, we have transformed them to a representation system proposed in Refs. 10, 11. We refer to this system as G94. All the above-mentioned sets of cmf's take positive values in G94. Tristimulus values, $\mathbf{X}$, in this system are obtained from those in the CIE 1931 system, $\mathbf{X}^{\prime}$, by introducing the matrix

$$
C_{X Y Z 31}^{G 94}=\left(\begin{array}{ccc}
0.9980 & 0.0020 & 0.0000 \\
0.0000 & 1.0000 & 0.0000 \\
0.2270 & -0.0428 & 0.8158
\end{array}\right),
$$

in expression (2). ${ }^{10,11}$ All the results obtained in this work are specified in G94.

The reference color stimuli used in this study, labeled A-E, are those suggested by the CIE. ${ }^{17}$ The tristimulus values associated with these stimuli are given in Table I. For 
TABLE I. Tristimulus values corresponding to the color centers recommended by the CIE. ${ }^{17}$ These data are expressed in the CIE 1931 (labeled with primes) and in the G94 color-representation systems.

\begin{tabular}{lcccrr}
\hline COLOR CENTER & $X_{1}^{\prime}$ & $X_{2}^{\prime}$ & $X_{3}^{\prime}$ & $X_{1}$ & $X_{2}$ \\
\hline A (achromatic) & 28.459 & 30.000 & 32.175 & 28.462 & 30.000 \\
B (red) & 19.954 & 14.100 & 7.174 & 19.942 & 14.100 \\
C (yellow) & 62.823 & 69.300 & 29.793 & 62.836 & 69.300 \\
D (green) & 16.442 & 24.000 & 25.856 & 16.457 & 9.778 \\
E (blue) & 8.922 & 8.800 & 23.0185 & 8.922 & 35.600 \\
\hline
\end{tabular}

the $l$-th color center we have produced a certain number $n_{l}$ of spectral distributions in the spectral range $I=[400$, 700] $\mathrm{nm}$ with a sampling interval of $\Delta \lambda=10 \mathrm{~nm}$. These distributions are metameric for the CIE 1931 standard observer. These procedure has been repeated for the different reference stimuli.

Note that there are several methods to produce metamers. In this study we have used a method developed by us. ${ }^{18,19} \mathrm{In}$ brief, this method uses a linear-programming technique based in the simplex algorithm. ${ }^{20}$ By taking into account physical color properties, such as excitation purity and dominant wavelength, an "objective function" is defined in terms of cmf's, and certain inequalities for the unknown spectral distributions $\rho_{j}^{l}(\lambda)$ are proposed. The key of the method is that these inequalities are given in terms of certain bounds, $V_{j}$, for the values of the unknown spectral distributions, i.e., $0<\rho_{j}^{l}\left(\lambda_{m}\right)<V_{j}$, where $\rho_{j}^{l}\left(\lambda_{m}\right)$ is the value of the spectral distribution sampling at point $\lambda_{m}$. By maximizing the objective function, we obtain the metamers. The algorithm was complemented with criteria of softness ${ }^{19}$ to produce spectral distributions of any color stimulus. The bounds $V_{j}$ are produced in a random way, thus this method provides a large number of different metamers (see Refs. 18, 19 for more details).

In this case, integrals are replaced by sums in Eq. (3):

$$
X_{i, j}^{k, l}=K \sum_{m=1}^{N} x_{i}^{k}\left(\lambda_{m}\right) \rho_{j}^{l}\left(\lambda_{m}\right) \Delta \lambda,
$$

$N$ is the number of wavelengths in which functions $\rho_{j}^{l}(\lambda)$ have been sampled (see Refs. 18, 19).

In the following analysis we consider $Q=5$ observers, $R=5$ reference color stimuli, and $S=n_{l}$ metameric distributions for each reference stimulus. Note that the number $n_{l}$ depends on the center considered: $n_{A}=1225, n_{B}=$ $1143, n_{C}=875, n_{D}=1224$, and $n_{E}=700$. These numbers are different, because for some color centers the algorithm used to produce the metamers requires a large amount of computing time.

The color discrimination ellipses for the observers JAM and $\mathrm{CF}$ have been measured by Martínez ${ }^{10}$ and Martínez et al. ${ }^{11}$ around the five color centers suggested by the CIE. Thus, we can compare the clouds of chromaticity coordinates $x_{i, j}^{k, l}$ obtained for the above-mentioned sets of cmf's with the corresponding ellipses for JAM and CF. The results obtained for the five color centers are shown in Fig. 1.

Note that the location of the clouds of points for observer
VOS is nearer to the ellipses than in the case of the other observers. This effect is reproduced for all color centers. The chromaticity coordinates computed for all the sets of cmf's are outside the corresponding ellipses. The clouds of points associated with observers VOS and SBM are partially inside the ellipses for color center E. The data for observers JAM and MM are always far from the ellipses, although they are close to each other.

In Tables II and III, quotients (4) are listed for all observers with regard to the ellipses measured for JAM and CF. Note the low values obtained for observer VOS. In most cases, quantity $\Omega^{k, l}$ is greater than unity. This result seems to indicate that the colorimetric behavior of the real observers clearly departs from that of the reference observer.

We can consider $d_{t h}^{k, l}$ as the just noticeable difference in the direction determined by the straight line joining points $\mathbf{x}^{\mathbf{k}, \mathbf{l}}$ and $\mathbf{x}_{\mathbf{t h}}^{\mathbf{k}, \mathbf{l}}$. In this way, high values of $\Omega^{k, l}$ indicate that the color performances of the $k$-th observer and the standard one are very different when considering the $l$-th color center. Thus, from the previous analysis we can conclude that the colorimetric behavior of real observers cannot be approximated by that of the reference observer. These results are in good agreement with those provided in Ref. 5. Authors of this work report significant displacements of the center of the ellipses when several sets of cmf's were analyzed (see Table I in Ref. 5). Looking for a check of these displacements, these authors calculated the tristimulus values of a limited number of theoretical metameric spectral reflectances, and they found a significant spreading of the chromaticity coordinates for the observers under consideration. Our results also agree with those reported in the studies of interobserver variability by other methods. ${ }^{2,9,11}$

For a given observer, $k$, we compute the average $\Omega^{k}=$ $\sum_{l=1}^{5} \Omega^{k, l} / 5$. This parameter can be used to obtain a quantitative measure of the global discrepancy between the standard and the $k$-th observer. In this way, we can establish an order of likeliness among the different observers with regard to the standard one. The results are listed in Table IV when considering the ellipse associated with observer CF. The same order of likeness is obtained when using the ellipse of JAM. The results obtained by using this method are coincidental with those reported in Ref. 21.

\section{INTEROBSERVER VARIABILITY}

The results obtained in the previous section are a consequence of the differences of cmf's of a given observer with 

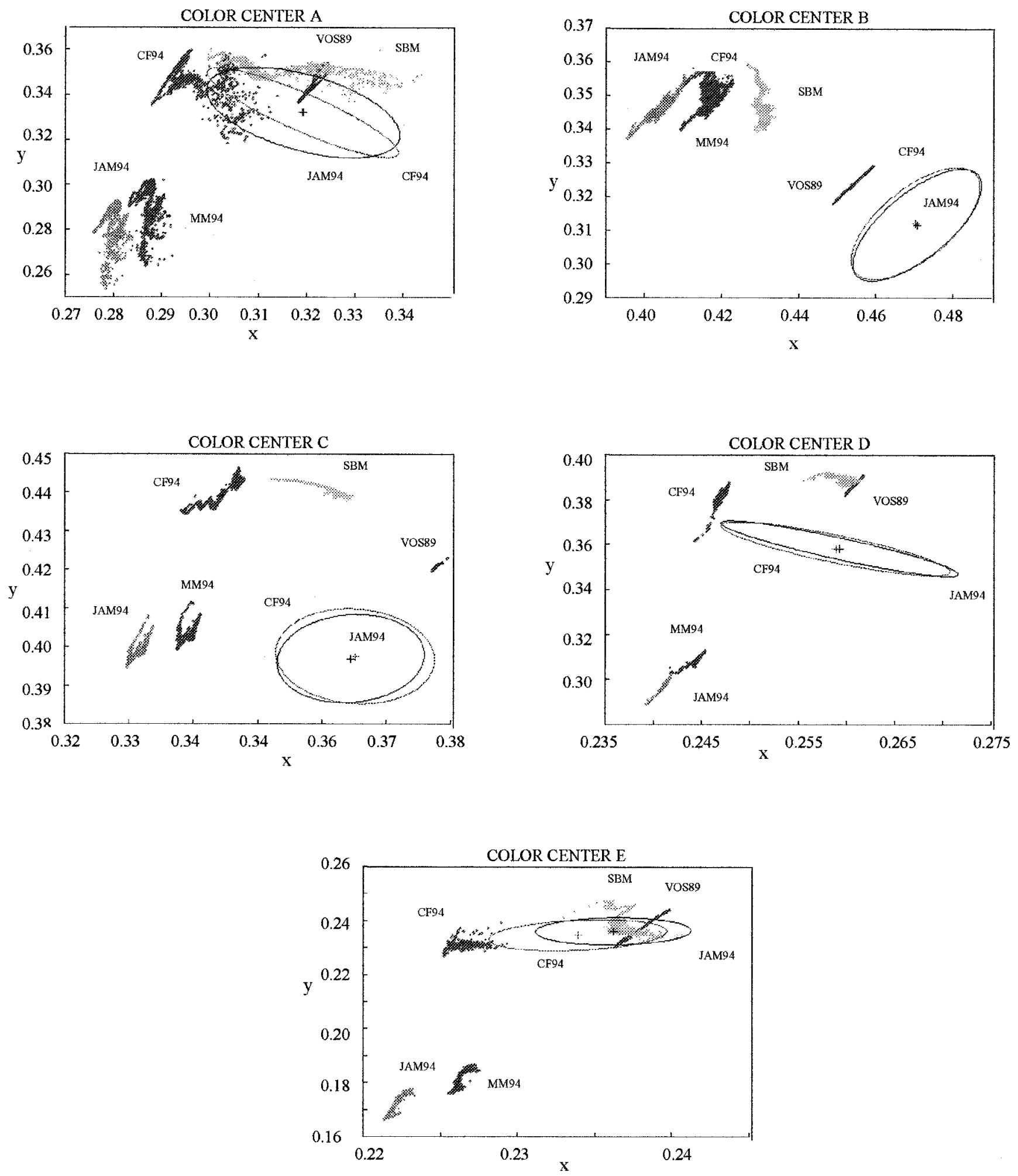

FIG. 1. Chromaticity coordinates obtained for each observer around the color centers studied. The data are represented in the chromaticity diagram associated to the CIE 1931 Standard Observer in the G94 system of primary stimuli.

regard to the cmf's of the standard observer. In this section, we intend to carry out a comparison restricted to observers JAM and CF. Martínez ${ }^{10}$ and Martínez et al. ${ }^{11,12}$ have measured the cmf's of the mentioned observers together with the thresholds corresponding to the five CIE centers. We analyzed the similarities of both observ- ers when perceiving these color centers. We used the following procedure:

1. For a given observer, JAM for example, and each color center $(l)$, we have produced a set of metameric spec- 
TABLE II. Quotients $\Omega^{k, l}$ computed for the specific observers with regard to the ellipse measured for CF.

\begin{tabular}{crrrrr}
\hline Color center & MM & CF & JAM & VOS & SBM \\
\hline A & 7.7 & 1.3 & 9.5 & 1.5 & 1.6 \\
B & 6.4 & 6.8 & 7.3 & 2.0 & 5.4 \\
C & 2.1 & 3.7 & 2.7 & 2.2 & 3.4 \\
D & 10.2 & 2.1 & 12.6 & 4.8 & 5.1 \\
E & 9.1 & 1.3 & 10.7 & 0.7 & 0.8 \\
\hline
\end{tabular}

tral distributions. The number of metamers is labeled as $n_{l}^{J A M}$ (see column 1 in Table V).

2. We computed the chromaticity coordinates for these distributions with cmf's associated with the other observer, CF in this case. The clouds of points together with the threshold determined for $\mathrm{CF}$ are represented in a common figure for each color center (see Fig. 2).

Steps 1 and 2 were repeated interchanging the role of the observers. The data for observer $\mathrm{CF}$ are given in column 2 of Table $\mathrm{V}$ and in Fig. 3.

Note in Fig. 2 that, for color centers $\mathrm{A}$ and $\mathrm{C}$, the points are partially inside the ellipse. In Fig. 3, the clouds of points are partially inside the ellipse when the color center A and $\mathrm{D}$ are considered; however, in the case of color center $\mathrm{B}$, all the points are inside the ellipse. The case is different for the rest of the color centers and each observer: the clouds of points are outside the ellipses. To quantify the discrepancy in evaluating the selected color centers, we have computed quotients $\Omega^{C F, l}$ (taking into account the results in Fig. 2) and $\Omega^{J A M, l}$ (taking into account the results in Fig. 3). These quotients are defined as in Eq. (4). The results are listed in columns 2 and 3 of Table VI, respectively.

When comparing results shown in Figs. 2 and 3, and data listed in Table VI, we deduce that color perception of observers JAM and CF clearly differs for color centers B, C, D, and E. In the case of the achromatic stimulus, A, the clouds of chromaticity coordinates are inside the corresponding ellipses.

This analysis reveals an important discrepancy between $\mathrm{CF}$ and JAM in evaluating color centers. We deduce that cmf's of the observers have a strong influence on the specification of individual colors. Note that according to Refs. 5, 12 , the variability appearing in the threshold parameters is not significant in most cases, although there was significant displacement of the ellipses' centers. In summary, the analysis reveals pronounced discrepancies between the two ob-

TABLE III. Quotients $\Omega^{k, l}$ computed for the specific observers with regard to the ellipse measured for JAM.

\begin{tabular}{crrrrr}
\hline Color center & MM & CF & JAM & VOS & SBM \\
\hline A & 3.6 & 1.1 & 4.4 & 0.7 & 1.0 \\
B & 7.0 & 7.5 & 8.0 & 2.2 & 5.9 \\
C & 2.4 & 4.3 & 2.9 & 2.3 & 3.8 \\
D & 13.0 & 2.3 & 16.1 & 5.9 & 6.4 \\
E & 10.7 & 2.1 & 12.7 & 0.3 & 0.5 \\
\hline
\end{tabular}

TABLE IV. Quantity $\Omega^{k}$ for the different observers when considering the threshold of CF. The pair of observers are ordered by their degree of global likeness with regard to CIE 1931 Standard Observer.

\begin{tabular}{lcc}
\hline $\begin{array}{c}\text { Pair of } \\
\text { observers }\end{array}$ & $\Omega^{k}$ & Order \\
\hline CIE31-VOS & 2.24 & 1 \\
CIE31-CF & 3.04 & 2 \\
CIE31-SBM & 3.28 & 3 \\
CIE31-MM & 7.10 & 4 \\
CIE31-JAM & 8.56 & 5 \\
\hline
\end{tabular}

servers evaluating a particular color, but strong similarities judging color differences.

Note that the results presented in Figs. 2 and 3, and Table VI reveal a nonreciprocity behavior between CF and JAM, except for color center A. This fact requires a brief description: the metamers generated for one observer, JAM for example, produce the same color perception for this observer, whereas the other observer, $\mathrm{CF}$ in this case, specifies each metamer as a different color. The quotients $\Omega^{C F, l}$ are estimators for the differences in the specification of the physical stimuli. This behavior is reproduced when the roles of the observers are interchanged. The surprising result is that quotients $\Omega^{C F, l}$ and $\Omega^{J A M, l}$ are clearly different between them for a given color center $l$, except for color centers A and C. This is because the color representation systems spanned by two different sets of cmf's are not isomorphic (see Section II), i.e., the correspondence between $R^{C F}$ and $R^{J A M}$ is not one-to-one: this point has been treated extensively in Refs. 22, 23. The origin of this behavior comes from the local differences in cmf's of different observers. In Ref. 6, a detailed statistical analysis of the cmf's of several observers was carried out. These authors introduced a parameter labeled as VAF (see the section entitled "Overall analysis of the cmf curve shape"), to test the similarity between two sets of cmf's. In Table IV of Ref. 6, it is shown how the VAF parameter for $\bar{x}_{\lambda}$ and $\bar{z}_{\lambda}$ are lower than the value for $\bar{y}_{\lambda}$. This fact confirms the nonisomorphic character between the $R^{C F}$ and $R^{J A M}$ color-representation systems.

\section{CONCLUSIONS}

From the analysis of data shown in Fig. 1 and Tables II and III, it can be concluded that the specification of a given physical stimulus strongly depends on the set of colormatching functions used. The clouds of points of chroma-

TABLE V. The number of metamers produced for each color center and observers JAM (column 2) and CF (column 3).

\begin{tabular}{crr}
\hline Color center & $n_{l}^{J A M}$ & $n_{l}^{C F}$ \\
\hline A & 1225 & 1225 \\
B & 964 & 992 \\
C & 1225 & 1225 \\
D & 1134 & 1225 \\
E & 1225 & 1225 \\
\hline
\end{tabular}



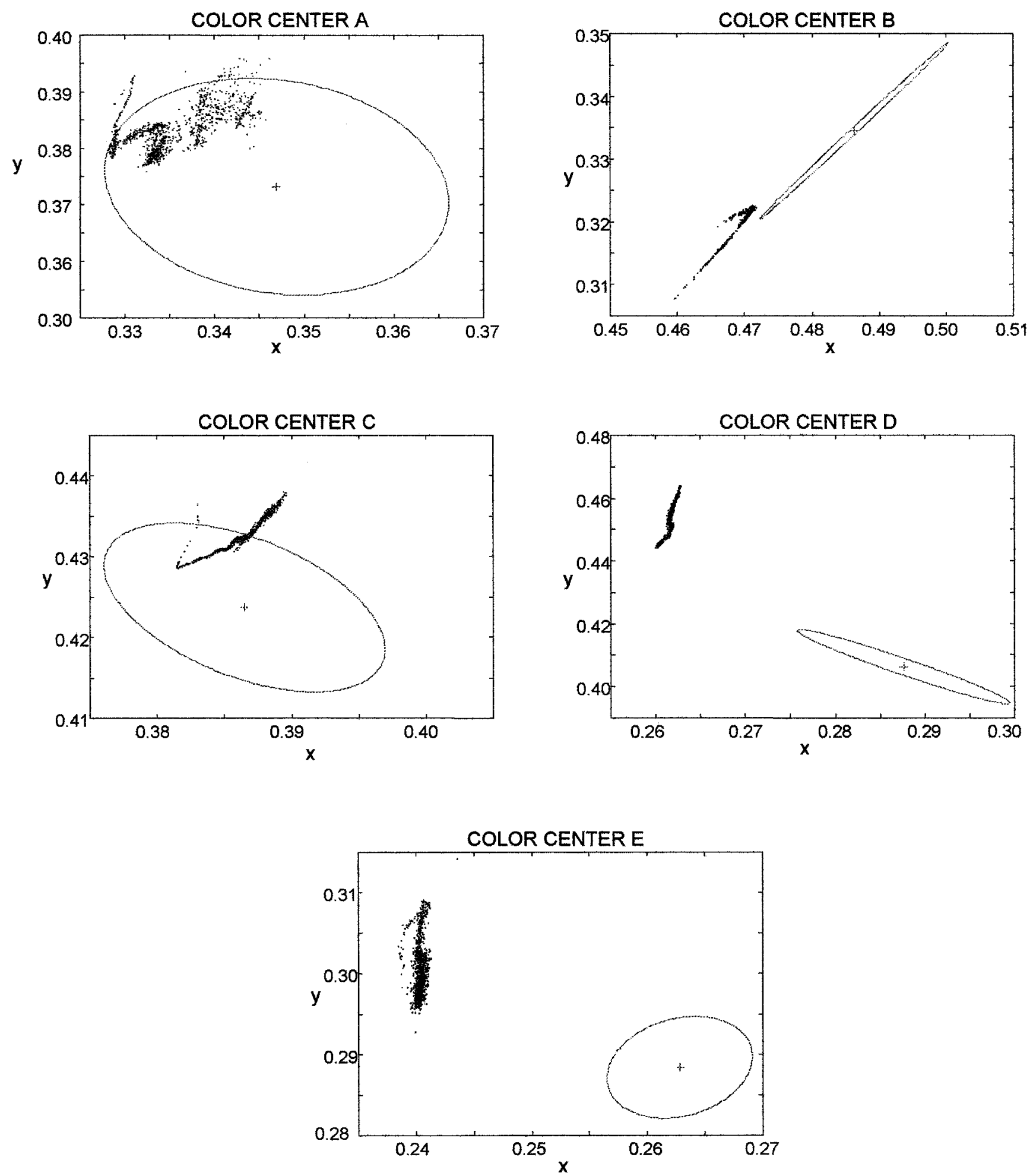

FIG. 2. Chromaticity coordinates obtained for observer CF for those distributions that are metamers for JAM. The continuous line represents the ellipse of CF for each color center. The results are referred to the G94 system.

ticity coordinates tell us that color perception of real observers is different from that of the standard observer when specifying a certain stimulus. Thus, real observers are not well represented by the set of cmf's of the standard observer. These results are in agreement with those reported in Refs. 6, 11, 12. In Ref. 3, the authors reported similar results concerning the displacement of the centers of the thresholds when considering isomeric and metameric color-matchings. They took this fact to be an indication of the failure in the colorimetric additivity. In our opinion this "failure" is a consequence of the nonisomorphic character between the color-representation system associated with each observer (see comments that follow).

Quantity $\Omega^{k}$ provides a quantitative measure of the dif- 

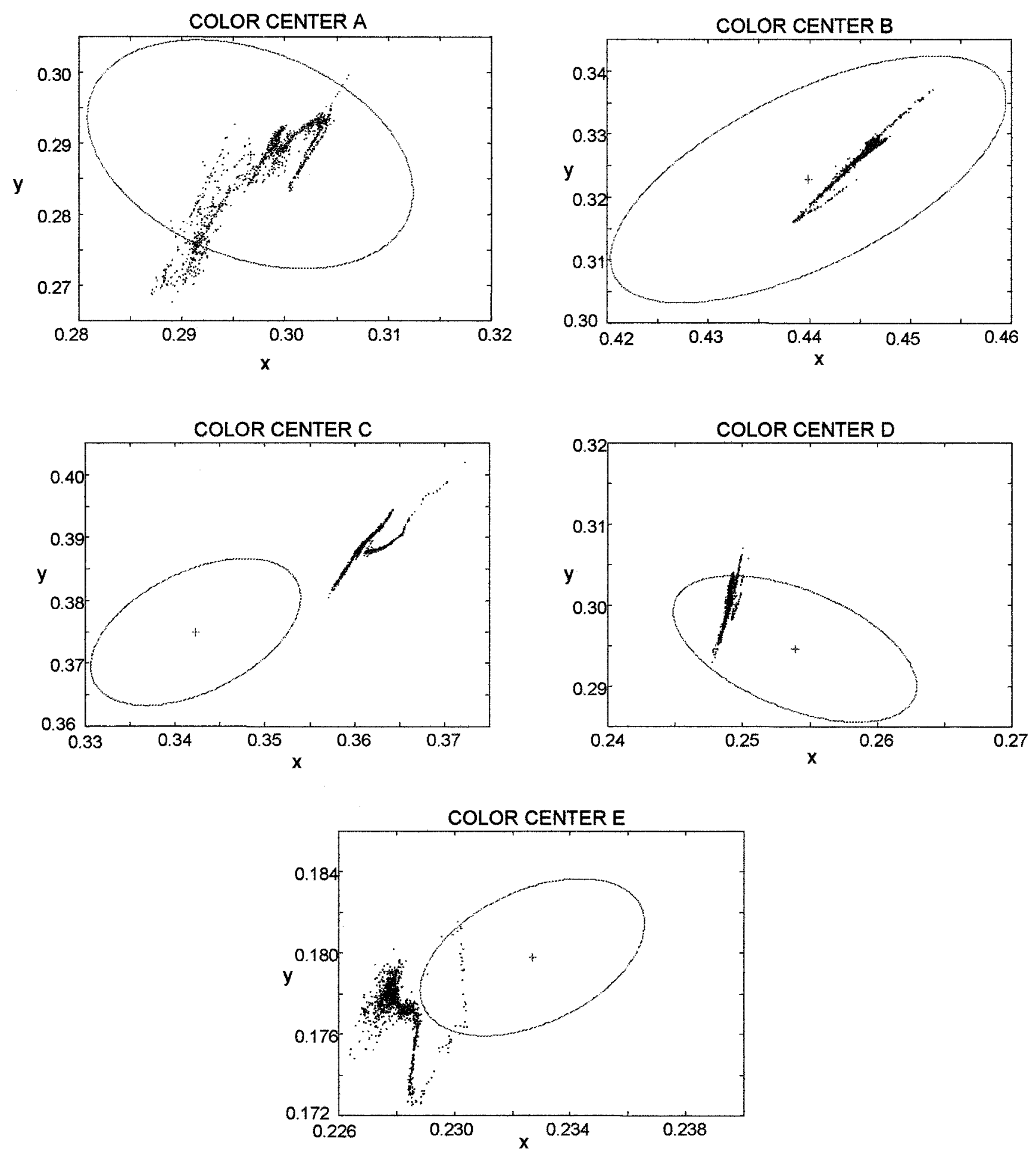

FIG. 3. Chromaticity coordinates obtained for observer JAM for those distributions that are metamers for CF. The continuous line represents the ellipse of JAM for each color center. The results are referred to the G94 system.

ference in the global colorimetric behavior of two observers. When comparing a set of several observers, this magnitude can be used to establish an order of likeness among them. The results listed in Table III are in accordance with those provided in Ref. 21. It has been shown in Section IV how there is no reciprocity between the colorimetric behavior predicted by the sets of cmf's associated with two real observers.
The problem underlying interobserver variability when comparing different observers was discussed in an article by Hita et al. ${ }^{14}$ In that work the causes of some anomalies found when representing the chromaticity thresholds in CIE 1931 chromaticity diagram were analyzed. These anomalies were considered to be a consequence of the distortion introduced when one set of experimental data is transformed from one color-representation system to another. To mini- 
TABLE VI. Quantities $\Omega^{C F, I}$ and $\Omega^{J A M, I}$. See text for details.

\begin{tabular}{ccc} 
Color center & $\Omega^{C F, I}$ & $\Omega^{J A M, I}$ \\
\hline A & 0.8 & 0.2 \\
B & 4.1 & 0.2 \\
C & 1.0 & 1.7 \\
D & 7.8 & 0.7 \\
E & 4.6 & 1.3 \\
\hline
\end{tabular}

mize the distortion, Hita et al. proposed to work with a unique system of primaries and with the same experimental dispositive. This is a partial solution to the problem derived from the nonisomorphic character between the color-representation systems associated with a given pair of observers. $^{22,23}$ This question together with the validity of establishing an adequate standard observer should be further investigated.

\section{ACKNOWLEDGMENTS}

The authors are especially endebted to Dr. J. A. Martínez, Dr. E. Hita, and Dr. M. Melgosa at Departamento de Optica of Granada University for their continual advice and help. They also want to express their gratitude to the anonymous referees who comments helped us to improve the article.

1. Judd DB. The color perception of deuteranopic and protanopic observers. J Opt Soc Am 1949;39:252-256.

2. North AD, Fairchild MD. Measuring color-matching functions. Part II. New data for assessing observer metamerism. Color Res Appl 1993; 18:163-170.

3. Hita E, Jiménez del Barco L, Romero J. Differential color thresholds from metameric matches: experimental results concerning failures of colorimetric additivity. J Opt Soc Am A 1986;3:1203-1209.

4. Jiménez del Barco L, Hita E, Jiménez JR, Romero J. Differential color thresholds and new results on failures of chromaticity and luminance prediction. J Opt 1994;25:81-92.

5. Melgosa M, Martínez JA, Pérez MM, Hita E. Comparative analysis of the performance of several color-matching functions proposed for small-size fields. Atti della Fondazione Giorgio Ronchi 1994;49:917931.

6. Pérez-Ocón F, Hita E, Jiménez del Barco L, Nieves JL. Contribution to the experimental review of the colorimetric standard observer. Color Res Appl 1999;24:377-388.

7. Stiles WS, Wyszecki G. Field trials of color-mixture functions. J Opt Soc Am 1962;52:58-75.

8. Viénot F. New equipment for the measurement of color-matching functions. Color Res Appl 1977;2:165-170.

9. Viénot F. Relation between inter- and intra-individual variability of color-matching functions. Experimental results. J Opt Soc Am 1980; 70:1476-1483.

10. Martínez JA. Estudio de la influencia de las funciones de mezcla sobre la determinación de diferencias de color. Ph.D. thesis, Univ Granada, Granada, 1995.

11. Martínez JA, Melgosa M, Hita E. Experimental determination of the color-matching functions of real observers. Comparative analysis of variability. Atti della Fondazione Giorgio Ronchi 1996;51:483-496.

12. Martínez JA, Poza AJ, Melgosa M, Hita E. Influence of colourmatching functions on threshold and large colour differences. J Opt 1997;28:26-36.

13. Wyszecki G, Stiles WS. Color science: concepts and methods, quantitative data and formulae. 2nd Ed. New York: Wiley; 1982.

14. Hita E, Alvarez-Claro M, Guerrero M. Differential thresholds representation of color. Atti della Fondazione Giorgio Ronchi 1977;32:882893.

15. Trezona PW. Individual observer data for the 1955 Stiles-Burch $2^{\circ}$ pilot investigation. J Opt Soc Am A 1987;4:769-782.

16. Vos JJ. Colorimetric and photometric of a $2^{\circ}$ fundamental observer. Color Res Appl 1978;3:125-128.

17. Robertson AR. CIE guidelines for coordinated research on colourdifference evaluation. Color Res Appl 1978;3:149-151.

18. Zoido JM, Carreño F, Bernabeu E. Improved linear programming method to generate metameric spectral distributions. Appl Opt 1995; 34:1938-1943.

19. Ezquerro JM, Zoido JM, Carreño F, Bernabeu E. Técnicas de suavizado aplicadas a la generación de distribuciones de potencia espectral radiante metaméricas. Optica Pura y Aplicada 1997;30:67-74.

20. Wilkinson JH, Reinsch C. Linear algebra. Vol. 2. In: Handbook for automatic computation, New York: Springer; 1971.

21. Zoido JM, Carreño F, Bernabeu E. The characteristic matrix of a colour detection system. J Opt A Pure Appl Opt 1999;1:371-377.

22. Zoido JM. Distancia estadística generalizada. La métrica del color. Ph.D. thesis. Universidad Complutense de Madrid, 1997.

23. Zoido JM. Optimization of color-representation systems when comparing different observers. Color Res Appl to appear. 